\title{
Economic Impact of Acinetobacter baumannii Infection in the Intensive Care Unit
}

\author{
Bruce Y. Lee, MD, MBA, Sarah M. McGlone, MPH, Yohei Doi, MD, PhD, Rachel R. Bailey, \\ $\mathrm{MPH}$, and Lee H. Harrison, MD \\ Public Health Computational and Operations Research (B.Y.L., S.M.M., R.R.B.), the Department \\ of Biomedical Informatics (B.Y.L., S.M.M., R.R.B.), and the Division of Infectious Diseases (Y.D., \\ L.M.H.), School of Medicine, and the Department of Epidemiology (B.Y.L., S.M.M., R.R.B.), \\ Graduate School of Public Health, University of Pittsburgh, Pittsburgh, Pennsylvania
}

\begin{abstract}
Although the prevalence of Acinetobacter baumannii infection appears to have been increasing over the past decade, its economic impact remains unclear. ${ }^{1,2} A$. baumannii infection tends to affect critically ill patients, causing serious infections in the intensive care unit (ICU), potentially increasing hospital lengths of stay (LOS) and mortality rates., ${ }^{2,3}$ Better understanding the economic effects of $A$. baumannii infection may help policy makers, hospital administrators, infection control professionals, and other healthcare workers determine how much to invest in interventions that can detect and control its spread.
\end{abstract}

Using TreeAge Pro 2009 (TreeAge Software), we developed a stochastic decision analytic computer simulation model that determined additional costs associated with $A$. baumannii in the ICU from the hospital perspective. An extended LOS associated with $A$. baumannii infection resulted in a loss of a hospital bed that could have been used by other patients and in corresponding lost revenues. Our model compared an ICU patient colonized with $A$. baumannii with a patient who was not colonized. Each colonized patient then had a probability of remaining simply colonized or developing an active $A$. baumannii infection, resulting in increased LOS and increased mortality. On the basis of findings from our search of the literature, we determined that colonization without infection did not affect a patient's LOS.

The model drew its clinical probabilities from the results of an extensive review of the literature. Our literature search identified all articles published from 1990 to the present in the Medline database using various combinations of the following key words: "acinetobacter," "infections," "prevalence," "multi-drug resistant," "nosocomial infections," "ICU," "colonization," "mortality," "length of stay," "economics," and "costs." We searched references from all relevant articles to identify additional studies. We reviewed and selected studies on the basis of the following inclusion and exclusion criteria: studies (largely case-control and cohort studies) were included if they included control subjects who were adequately matched on the basis of severity of illness and comorbidities (using measures such as Acute Physiology and Chronic Health Evaluation [APACHE] score, the McCabe score, and the Charlson comorbidity index), clearly identified and characterized the study population, and reported clinical outcomes (eg, mortality and LOS). A total of 5

(C) 2010 by The Society for Healthcare Epidemiology of America. All rights reserved.

Address reprint requests to Bruce Y. Lee, MD, MBA, Public Health Computational and Operations Research (PHICOR), University of Pittsburgh, 200 Meyran Avenue, Suite 200, Pittsburgh, PA 15213 (BYL1@ pitt.edu).

Potential conflicts of interest. All authors report no conflicts of interest relevant to this article. 
studies reported the additional LOS after the infection event. Studies without human subjects or matching control subjects were excluded.

For our baseline scenario, patients with active $A$. baumannii infection had an additional LOS drawn from a $\gamma$ distribution (mean LOS \pm standard deviation, $25.23 \pm 10.59$ days). ${ }^{1,3-6}$ This distribution represents the mean and standard deviation of all reported mean attributable LOSs from the studies identified by our literature review. The $\gamma$ distribution models continuous variables that are always positive and have a skewed distribution, with a long upper tail representing patients with long LOSs. Those without active $A$. baumannii infection (ie, persons who were colonized but not infected) had an LOS of 0.66 times that of persons with active infection. (Sensitivity analyses varied the attributable LOS). ${ }^{1-3,7}$ The cost per bed-day was $\$ 4,397.50$ (triangular distribution; range, $\$ 1,000-\$ 8,000$ ) on the basis of mean daily costs for patients in the ICU who underwent ventilation and those who did not. $^{8}$ All costs were in 2010 US dollars, with a 3\% discount rate used to convert costs from other years. We assumed that $20 \%-70 \%$ of Acinetobacter-colonized patients developed infection.

Each simulation run sent 1,000 simulated ICU patients 1,000 times (a total of 1,000,000 trials) through the model. Table 1 shows how per-patient $A$. baumannii-attributable costs increased as the proportion of patients with $A$. baumannii who have active infection increased. For a $20 \%$ infection probability, the mean cost to a hospital of each $A$. baumannii case ( \pm standard deviation) was $\$ 8,246 \pm \$ 4,472$. Increasing the proportion of infections to $70 \%$ increased the cost to the hospital to $\$ 29,019 \pm \$ 15,977$. Table 1 also lists results from sensitivity analyses ranging attributable LOS from infection and how cost to the hospital changed with the number of $A$. baumannii cases per month.

These numbers could confer a considerable economic burden to hospitals. For example, over a 6-month period in 2008 in a University of Pittsburgh Medical Center hospital, 25 of 626 ICU-admitted patients were colonized with the organism (prevalence, $4 \%$ ), translating to a cost to the hospital of $\$ 412,291-\$ 1,621,199$ for the year. During the period 2006-2007, 463 hospitals reported healthcare-associated infections to the National Healthcare Safety Network. ${ }^{9}$ Of 28,502 reported infections, A. baumannii caused 902 (2.7\%), resulting in costs ranging from $\$ 7.4$ million to $\$ 26.1$ million.

To our knowledge, our study is the first to use economic modeling to quantify the economic burden of $A$. baumannii to hospitals. Understanding costs from the hospital perspective is important, because it may help hospitals determine how much should be invested in infection control to prevent the spread of this organism. We demonstrated that, even with a conservative estimate of the proportion of colonizations to develop into infection (at least $20 \%$ ), the financial burden to hospitals can be substantial. The ratio of infection to colonization may vary widely depending on the patient population. Studies from the literature have reported infection rates of $32.4 \%, 55 \%,{ }^{10}$ and $64 \% .{ }^{7}$ Therefore, it is possible that the actual financial impact of $A$. baumannii colonization is closer to the upper end of our estimate (ie, approximately $50 \%$ of colonizations representing infection). Even low $A$. baumannii prevalence can be a significant burden to a hospital, suggesting that hospitals may consider further investigation into controlling this infectious pathogen. Our model may, in fact, underestimate the cost of $A$. baumannii colonization and infection, because it only considered lost bed-days and did not include additional costs associated with infection, such as treatment, additional surgery, and ventilator use. Attributing such treatment costs is difficult, because the patients tend to be very ill and to have multiple comorbidities.

Key limitations of our study include the facts that models simplify real life, cannot fully represent every event or outcome or the heterogeneity of patient populations, and draw 
disparate data from studies of varying quality. The studies from our literature review were limited, because it is difficult to perform outcome studies of antibiotic resistance that truly control for severity of illness and to determine attributable increased LOS.

Individual hospitals may want to use the results of our model to determine the economic burden of $A$. baumannii in their specific hospitals, given their unique circumstances. Additional research into the probability of infection and potential control measures may be warranted.

\section{Acknowledgments}

Financial support. The National Institute General Medical Sciences Models of Infectious Agent Study (1U54GM088491-0109) and the Pennsylvania Department of Health.

\section{References}

1. Abbo A, Carmeli Y, Navon-Venezia S, Siegman-Igra Y, Schwaber MJ. Impact of multidrugresistant Acinetobacter baumanii on clinical outcomes. Eur J Clin Microbiol Infect Dis. 2007; 26:793-800. [PubMed: 17701063]

2. Lee N-Y, Lee H-C, Ko N-Y, et al. Clinical and economic impact of multidrug resistance in nosocomial Acinetobacter baumannii bacteremia. Infect Control Hosp Epidemiol. 2007; 28(6):713719. [PubMed: 17520546]

3. Grupper M, Sprecher H, Mashiach T, Finkelstein R. Attributable mortality of nosocomial Acinetobacter bacteremia. Infect Control Hosp Epidemiol. 2007; 28(3):293-298. [PubMed: 17326019]

4. Sunenshine RH, Wright M-O, Maragakis LL, et al. Multidrug-resistant Acinetobacter infection mortality rate and length of hospitalization. Emerg Infect Dis. 2007; 13(1):97-103. [PubMed: 17370521]

5. Weingarten CM, Rybak MJ, Jahns BE, Stevenson JG, Brown WJ, Levine DP. Evaluation of Acinetobacter baumannii infection and colonization, and antimicrobial treatment patterns in an urban teaching hospital. Pharmacotherapy. 1999; 19(9):1080-1085. [PubMed: 10610015]

6. The Brooklyn Antibiotic Resistance Task Force. The cost of antibiotic resistance: effect of resistance among Staphylococcus aureus, Klebsiella pneumoniae, Acinetobacter baumannii, and Pseudomonas aeruginosa on length of hospital stay. Infect Control Hosp Epidemiol. 2002; 23:106108. [PubMed: 11893146]

7. Garcia-Garmendia J-L, Ortiz-Leyba C, Garnacho-Montero J, Jimenez-Jimenez F-J, MonterrubioVillar J, Gili-Miner M. Mortality and the increase in length of stay attributable to the acquisition of Acinetobacter in critically ill patients. Crit Care Med. 1999; 27(9):1794-1799. [PubMed: 10507600]

8. Cooke CR, Kahn JM, Watkins TR, Hudson LD, Rubenfeld GD. Cost-effectiveness of implementing low-tidal volume ventilation in patients with acute lung injury. Chest. 2009; 136:79-88. [PubMed: 19318673]

9. Hidron AI, Edwards JR, Patel J, et al. Antimicrobial-resistant pathogens associated with healthcareassociated infections: annual summary of data reported to the National Healthcare Safety Network at the Centers for Disease Control and Prevention, 2006-2007. Infect Control Hosp Epidemiol. 2008; 29(11):996-1011. [PubMed: 18947320]

10. Rodríguez-Baño J, García L, Ramírez E, et al. Long-term control of hospital-wide, endemic multidrug-resistant Acinetobacter baumannii through a comprehensive "bundle" approach. Am J Infect Control. 2009; 37(9):715-722. [PubMed: 19457584] 
TABLE 1

Cost of Cases of Acinetobacter Colonization to the Hospital

\begin{tabular}{|c|c|c|c|c|}
\hline \multirow{2}{*}{$\begin{array}{l}\text { Proportion of subjects with active } \\
\text { infection }\end{array}$} & \multirow[b]{2}{*}{ Cost per case, ${ }^{a}$ US\$, mean \pm SD } & \multicolumn{3}{|c|}{ Cost per year, US\$, mean \pm SD } \\
\hline & & 1 Case/mo & 2 Cases/mo & 3 Cases $/ \mathrm{mo}$ \\
\hline \multicolumn{5}{|c|}{ Baseline distribution of attributable $\operatorname{LOS}^{b}$} \\
\hline $20 \%$ & $8,246 \pm 4,472$ & $98,950 \pm 15,491$ & $197,900 \pm 21,908$ & $296,849 \pm 26,831$ \\
\hline $30 \%$ & $12,490 \pm 6,926$ & $149,884 \pm 23,994$ & $299,797 \pm 33,933$ & $449,651 \pm 41,559$ \\
\hline $40 \%$ & $16,072 \pm 8,870$ & $192,866 \pm 30,727$ & $385,732 \pm 43,455$ & $578,598 \pm 53,221$ \\
\hline $50 \%$ & $19,731 \pm 10,403$ & $236,768 \pm 36,036$ & $473,536 \pm 50,962$ & $710,304 \pm 62,415$ \\
\hline $60 \%$ & $24,805 \pm 12,956$ & $297,656 \pm 44,882$ & $595,312 \pm 63,473$ & $348,231 \pm 77,739$ \\
\hline $70 \%$ & $29,019 \pm 15,977$ & $348,231 \pm 55,345$ & $696,462 \pm 78,269$ & $1,044,692 \pm 95,860$ \\
\hline \multicolumn{5}{|l|}{5 Days attributable LOS } \\
\hline $20 \%$ & $4,738 \pm 1,567$ & $56,857 \pm 18,800$ & $113,714 \pm 37,599$ & $170,571 \pm 56,399$ \\
\hline $30 \%$ & $7,211 \pm 2,264$ & $86,536 \pm 27,168$ & $173,072 \pm 54,337$ & $259,608 \pm 81,505$ \\
\hline $40 \%$ & $9,431 \pm 3,040$ & $113,176 \pm 26,481$ & $226,352 \pm 72,963$ & $339,527 \pm 109,444$ \\
\hline $50 \%$ & $11,777 \pm 3,817$ & $141,323 \pm 45,802$ & $282,645 \pm 91,604$ & $423,968 \pm 137,406$ \\
\hline $60 \%$ & $14,412 \pm 4,589$ & $172,942 \pm 56,269$ & $345,883 \pm 112,537$ & $518,825 \pm 168,806$ \\
\hline $70 \%$ & $16,895 \pm 5,199$ & $202,743 \pm 62,374$ & $405,486 \pm 124,748$ & $608,228 \pm 187,122$ \\
\hline \multicolumn{5}{|l|}{10 Days attributable LOS } \\
\hline $20 \%$ & $9,523 \pm 3,062$ & $114,274 \pm 36,739$ & $228,547 \pm 73,479$ & $342,821 \pm 110,218$ \\
\hline $30 \%$ & $14,207 \pm 4,617$ & $170,484 \pm 55,401$ & $340,968 \pm 110,802$ & $511,452 \pm 166,203$ \\
\hline $40 \%$ & $19,114 \pm 6,289$ & $229,364 \pm 75,470$ & $458,728 \pm 150,940$ & $688,092 \pm 226,410$ \\
\hline $50 \%$ & $24,038 \pm 7,802$ & $288,460 \pm 93,619$ & $576,921 \pm 187,239$ & $865,381 \pm 280,858$ \\
\hline $60 \%$ & $28,407 \pm 9,158$ & $340,882 \pm 109,900$ & $681,765 \pm 219,800$ & $1,022,647 \pm 329,701$ \\
\hline $70 \%$ & $33,003 \pm 10,292$ & $396,040 \pm 123,508$ & $792,080 \pm 247,016$ & $1,188,120 \pm 370,524$ \\
\hline \multicolumn{5}{|l|}{15 Days attributable LOS } \\
\hline $20 \%$ & $13,988 \pm 4,559$ & $167,855 \pm 54,710$ & $335,710 \pm 109,419$ & $503,565 \pm 164,129$ \\
\hline $30 \%$ & $21,245 \pm 6,928$ & $254,941 \pm 83,131$ & $509,883 \pm 166,262$ & $764,824 \pm 249,392$ \\
\hline $40 \%$ & $28,237 \pm 9,091$ & $338,844 \pm 109,094$ & $677,688 \pm 218,188$ & $1,016,532 \pm 327,281$ \\
\hline $50 \%$ & $35,312 \pm 11,212$ & $423,743 \pm 134,541$ & $847,485 \pm 269,082$ & $1,271,228 \pm 403,623$ \\
\hline $60 \%$ & $43,099 \pm 14,115$ & $517,183 \pm 169,381$ & $1,034,365 \pm 338,762$ & $1,551,548 \pm 508,142$ \\
\hline $70 \%$ & $49,608 \pm 16,429$ & $595,299 \pm 197,148$ & $1,190,599 \pm 394,295$ & $1,785,898 \pm 591,443$ \\
\hline
\end{tabular}

NOTE. LOS, length of stay; mo, month; SD, standard deviation.

${ }^{a}$ A case was defined as carriage of $A$. baumannii or active A. baumannii infection.

$b_{\text {Baseline, } 25.23 \pm 10.59 \text { days. }}$ 